\title{
Influencing factors of droplet aggregation on hierarchical wedge-shaped functional surfaces
}

\author{
Shuai Wang ${ }^{\mathrm{a}, \mathrm{d}}$, Zhilong Peng ${ }^{\mathrm{a}, \mathrm{d}}$, Jianjun $\mathrm{Li}^{\mathrm{b}}$, Yazheng Yang ${ }^{\mathrm{a}, \mathrm{d}}$, Chao Wang ${ }^{\mathrm{c}, \mathrm{e}, *}$, Shaohua Chen ${ }^{\mathrm{a}, \mathrm{d}, *}$ \\ ${ }^{\text {a } I n s t i t u t e ~ o f ~ A d v a n c e d ~ S t r u c t u r e ~ T e c h n o l o g y, ~ B e i j i n g ~ I n s t i t u t e ~ o f ~ T e c h n o l o g y, ~ B e i j i n g ~ 100081, ~ C h i n a ~}$ \\ ${ }^{\mathrm{b}}$ State Key Laboratory of High Performance Complex Manufacturing, College of Mechanical and Electrical Engineering, Central South University, Changsha 410083, \\ Hunan, China \\ ${ }^{\mathrm{c}}$ LNM, Institute of Mechanics, Chinese Academy of Sciences, Beijing 100190, China \\ ${ }^{\mathrm{d}}$ Beijing Key Laboratory of Lightweight Multi-functional Composite Materials and Structures, Beijing Institute of Technology, Beijing 100081, China \\ ${ }^{\mathrm{e}}$ School of Engineering Science, University of Chinese Academy of Sciences, Beijing 100049, China
}

\section{A R T I C L E I N F O}

\section{Keywords:}

Spontaneous droplet aggregation

Functional surface

Hierarchical wedge-shaped zone

Influencing factors

Water collection

\begin{abstract}
A B S T R A C T
Spontaneous droplet aggregation has great potential applications in liquid transportation, droplet-based microfluidics and water collection in deserts. Several novel hierarchical functional surfaces have been well prepared experimentally, on which small droplets would aggregate to form large droplets. The common feature of the hierarchical surface is wedge-shaped functional zone. However, how to guarantee the stability of water collecting function in real design and application is still lack of theoretical guidance. Based on molecular dynamics simulations, numerical experiments are systematically carried out in the present paper. Several important influencing factors of droplet spontaneous aggregation are studied. It is found that three typical sizedependent moving patterns of droplets may exist on such a hierarchical functional surface, i.e. lingering, pinning and directional transporting. The physical mechanism is further revealed for each pattern in terms of potential energy, which significantly depends on the wedge angle, direction of the wedge and symmetry of the bifurcation of each level. In order to achieve spontaneous droplet aggregation effectively, three conditions need to be met: a relatively small wedge angle at each level, wedges in the same direction at different levels, and asymmetric arrangement of wedges at the same level. A three-level functional surface is further numerically designed, which can realize water collection successfully. The results in this paper should be useful for the precise design of hierarchical functional surfaces of water collection in practical application.
\end{abstract}

\section{Introduction}

The spontaneous transportation and aggregation of droplets have attracted increasing attention in both scientific and engineering fields, such as liquid transportation [1], droplet-based microfluidics [2], labon-a-chip [3], atmospheric water collection [4,5] and so forth. Recent studies [6-9] on biological surfaces have already shown amazing water collecting abilities, which benefit from their heterogeneous wetting properties. For example, the back of a desert beetle [7] consists of separated no-waxy hydrophilic and wax-coated hydrophobic areas; Spider silk $[8,9]$ has a periodic structure comprising highly hydrophilic spindle-knots and hydrophobic joints.

Inspired by the natural design, various regular hydrophilic patterns of square [10], circle [11], star [12], line [13] and mesh [14] dispersedly on a hydrophobic substrate were printed to introduce "wettability gradient" [15]. On such surfaces, droplets near the boundary between the hydrophilic and hydrophobic regions would spontaneously move into the hydrophilic zone, which induces the droplets to grow $[16,17]$. Hydrophilic patterns with wedge-like shapes rather than regular circles or square spots were further designed to introduce the "shape gradient" [18-23]. On such surfaces, droplets can not only be agglomerated into the wedge-shaped hydrophilic regions but also be transported a long distance from the narrow end to the wide end in the hydrophilic band. Zheng et al. [24] and Zhang et al. [19] indicated that droplets on such a single wedge-like region were actuated by the unbalanced interfacial tension induced by both the wetting gradient and the shape gradient. Using the molecular dynamics simulation method, Wang et al. [25] gave an explanation for the droplet spontaneous transportation from the perspective of potential energy, in which surfaces with functions of droplet turning, pinning, accelerating,

\footnotetext{
*Corresponding authors at: LNM, Institute of Mechanics, Chinese Academy of Sciences, Beijing 100190, China (C. Wang). Institute of Advanced Structure Technology, Beijing Institute of Technology, Beijing 100081, China (S. Chen).

E-mail addresses: wangchao@lnm.imech.ac.cn (C. Wang), chenshaohua72@hotmail.com, shchen@bit.edu.cn (S. Chen).
} 
gathering and path selecting, were further designed numerically.

Based on the design of the single wedge-like hydrophilic region, a tree-like hierarchical wedge-shaped scheme was further well proposed [26-28] in order to realize water collection in practical applications $[4,29]$. Zheng et al. [24] fabricated a two-level wedge-shaped surface, on which droplets deposited onto the tip of hydrophilic wedge-like branches could spontaneously move to the main trunk effectively. Inspired by the leaf vein, Lin et al. [27] fabricated four-level wedgeshaped trunks on flexible hydrophobic polyethylene terephthalates, on which the water-collecting efficiency increases more than 10 times due to the shape gradient and gravity. Wang et al. [26] directly wrote a treeshaped hierarchical structure on a superhydrophobic film with laser and obtained an improved efficiency of water collection with a factor of about 36 compared with the superhydrophobic surface. Liu et al. [28] proposed a hybrid scheme to combine both regular mesh and irregular wedge-shaped trunk to obtain a higher coverage rate of hydrophilic region, and obtained a notable enhancement in water collection efficiency compared with the conventional superhydrophobic surfaces.

Although hierarchical wedge-shaped designs do enhance droplet aggregation in comparison with conventional surfaces, precise design of hierarchical wedge-shaped surfaces can ensure the stability of water collecting function in practical applications. Main factors that may influence droplet transportation and aggregation on a hierarchical wedgeshaped surface should be systematically studied, for example, the connecting topology of wedge-like branches, the size of each-level wedge region and the wedge angle, etc.

In order to investigate the influencing factors, numerical experiment is a better choice. Compared with a real experiment, numerical experiment is not only more convenient to change parameters, but also can give a reasonable influencing law. All-atom molecular dynamics simulation is adopted in this paper. Spontaneous transportation and aggregation of droplets on a hierarchical wedge-shaped functional surface are systematically studied. Both the influence of droplet volume and that of the hierarchical topology, such as the level number, wedge angle, wedge direction and symmetry of bifurcations, are investigated. All the results are explained by the method of potential energy comparison. This study should be helpful for the precise design of hierarchical functional surfaces of water collection in practical application.

\section{Simulation model and method}

As a representative numerical model, a two-level functional surface is shown in Fig. 1, in which Fig. 1a denotes the top view and Fig. 1b is the front view. The two-level functional surface is represented by a rectangle graphene sheet with a size of $30 \times 10 \mathrm{~nm}$, which is assumed to be rigid. The surface consists of a purple hydrophobic area and a yellow hydrophilic area as shown in Fig. 1a. The two-level functional surface refers to the hydrophilic regions, including a main trunk and four small branches. Both the main trunk and small branches have a

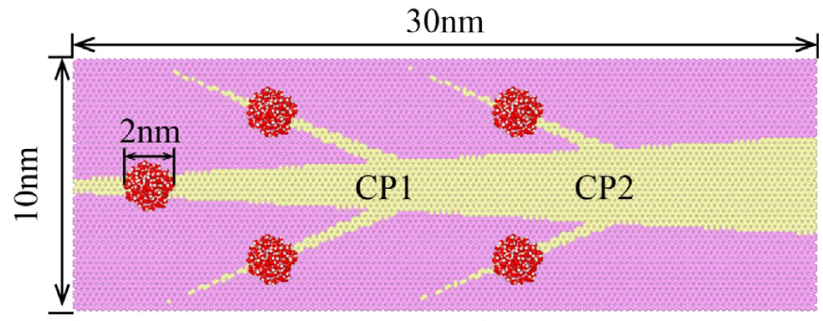

(a) top view

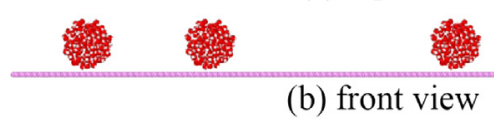

Fig. 1. Schematic of a two-level functional surface. (a) The top and (b) front view. wedge-like shape. The cross point (CP) between the main trunk and branches is numbered as $\mathrm{CP} 1$ and $\mathrm{CP} 2$ in this paper. Five water droplets are initially placed at the narrow ends of the main trunk and four branches.

The standard model (SPC/E) [30,31] with the SHAKE algorithm [32] is adopted to simulate the behavior of water droplets. The particleparticle particle-mesh (PPPM) algorithm [33] is adopted to achieve the long-range Coulomb interaction of droplets. Both the hydrophilic and hydrophobic regions on the substrate surface are characterized by Lennard-Jones potential $\varphi(r)=4 \varepsilon\left[(\sigma / r)^{12}-(\sigma / r)^{6}\right]$ with the same equilibrium distance of $\sigma=0.319 \mathrm{~nm}$, but different depths of energy well of $\varepsilon=5.85 \mathrm{meV}$ and $\varepsilon=1.95 \mathrm{meV}$, which have been widely used to study the wettability of surface $[25,34,35]$. Contact angles corresponding to the two depths of energy well $\varepsilon$ are $51^{\circ}$ and $138^{\circ}$, which can be actually achieved through different techniques in experiments, for examples, charge carrier density [36], oxygen-containing functional groups [37] or the hierarchically structure [38]. The droplets are initially kept at $300 \mathrm{~K}$ with Nosé-Hoover thermostats [39] for enough time to reach the corresponding thermal equilibrium and optimal configuration. Then, the droplets are placed at the narrow ends of the main trunk and branches. The cutoff distance is set to $1 \mathrm{~nm}$ and the time step is $1 \mathrm{fs}$ in all simulations. All simulations are implemented with an open source software Large-scale Atomic/Molecular Massively Parallel Simulator (LAMMPS). [40]

\section{Spontaneous droplet aggregation on a two-level functional surface}

As the treated droplets are placed at the narrow ends of the hydrophilic main trunk and branches, all droplets spontaneously move in the wider direction of the wedge. Snapshots are shown in Fig. 2. At the initial stage as shown in Fig. 2a, the droplet "1" on the narrow end of the hydrophilic main trunk moves towards the wider direction, as indicated by the arrow, while the other droplets " 2 ", " 3 ", " 4 " and " 5 " on the narrow ends of branches move towards the main trunk. The mechanism of these motions should be consistent with the case of a droplet on a single wedge, which abides by the principle of minimum potential energy [25]. At 0.06 ns, droplets " 4 " and "5" obtain a very high speed of about $128 \mathrm{~m} / \mathrm{s}$ and $101 \mathrm{~m} / \mathrm{s}$, respectively, and aggregate with each other to form a new droplet labeled as " $4 \sim 5$ " near CP2 as shown in Fig. 2b. The aggregated droplet " $4 \sim 5$ " keeps moving with a speed of about $106 \mathrm{~m} / \mathrm{s}$. Eventually, droplet " $4 \sim 5$ " stops at a certain point, where the driving force vanishes $[19,24,25]$. At the same time, droplets " 1 ", " 2 " and " 3 " move toward CP1 and aggregate to form a bigger droplet " $1 \sim 3$ " at about 0.1 ns as shown in Fig. 2c. The emerging droplet " $1 \sim 3$ " keeps moving forward. When the droplet " $1 \sim 3$ " reaches the place that the droplet " $4 \sim 5$ " stays, the two come together to form a larger droplet " $1 \sim 5$ " as shown in Fig. 2 d. At this time, the gradient wedge shape and the large size of droplet " $1 \sim 5$ " would induce a new driving force on the new droplet " $1 \sim 5$ ", causing the droplet to move further forward as shown in Fig. 2e. Here, it should be noted that the high moving velocity is a unique feature for the transportation of nano-objects $[25,35]$. The whole aggregation process is given by Movie S1 in Supporting Information. Similar spontaneous droplet aggregation has been well observed in experiments. For example, Wang et al. [26] showed that droplets condensed from fog could move along multiple wedge-shaped paths and gather into larger droplets. However, pinning behavior of droplets is widely observed in experiments, which is not conducive to water collection. How can we design a functional surface with high water collection efficiency? A systematic study of factors that affect the efficiency of water collection should be carried out. 

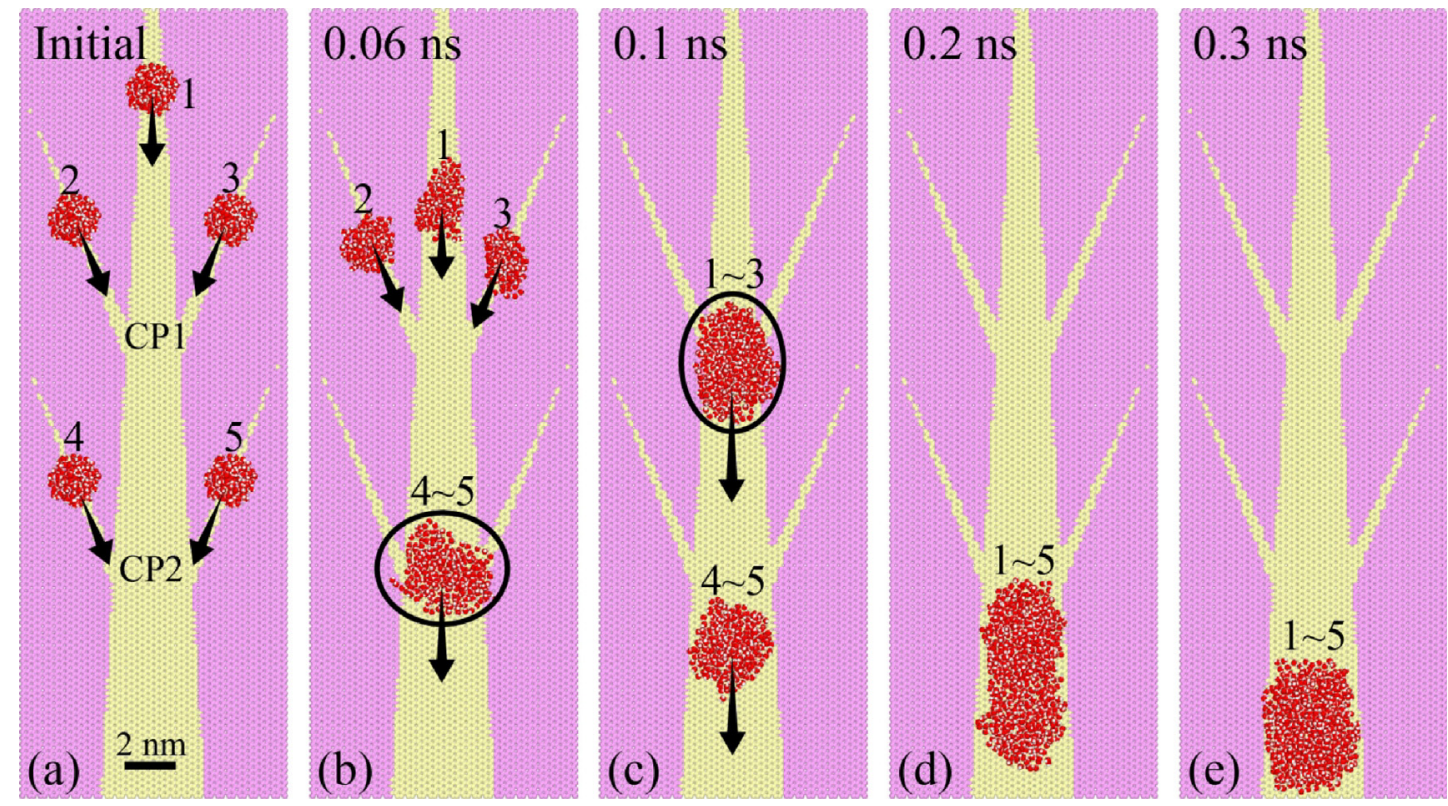

Fig. 2. Snapshots of droplets spontaneously moving on the surface.

\section{The mechanism of spontaneous droplet aggregation and its influencing factors}

In order to find the mechanism of multiple droplet aggregation on a hierarchical wedge-shaped functional surface, the transport behavior of a single droplet on such a surface with only a cross point is first studied. The width at the cross point is fixed as $W_{\mathrm{cp}}=2 \mathrm{~nm}$. Three droplets with a respective diameter $d$ of 1.5, 2.5, $5 \mathrm{~nm}$ are adopted, which are placed on the cross point of wedge-shaped hydrophilic main trunk, three typical moving behaviors are found. For the small droplet with a diameter $1.5 \mathrm{~nm}$, it will randomly linger between the cross point and the right region of the hydrophilic wedge-shaped zone and the arrow indicates the path of the droplet as shown in Fig. 3a1. For the medium sized droplet with a diameter $2.5 \mathrm{~nm}$, it will be pinned at the cross point as shown in Fig. 3a2. For the large droplet with a diameter $5 \mathrm{~nm}$, the droplet will pass through the cross point and keep moving toward the larger area of the hydrophilic wedge-shaped zone as shown in Fig. 3a3. Systematic simulation gives two critical ratios $\sim 1.25$ and $\sim 1.75$ of the droplet diameter to the cross point width $d / W_{\text {cp }}$ as shown in Fig. S1 in Supporting Information. The two values divide the behavior of droplets of different diameters into three typical cases, i.e., randomly lingering, pinning and directional moving, as shown in Movie S2 in Supporting Information. It means when the ratio of droplet diameter to the cross point width is less than 1.25 , droplets will linger on the functional surface similar to that shown in Fig. 3a1. When the ratio of droplet diameter to the cross point width is larger than 1.75, droplets will move directionally on the functional surface similar to that shown in Fig. 3a3. When this ratio is between 1.25 and 1.75, droplets will be pinned at the cross point as shown in Fig. 3a2. Similar behaviors of pinning and directional moving of droplets on hierarchical wedge-shaped functional surface were also be observed in experiments [24,26], while the random motion is not obvious in experiments due to the negligible
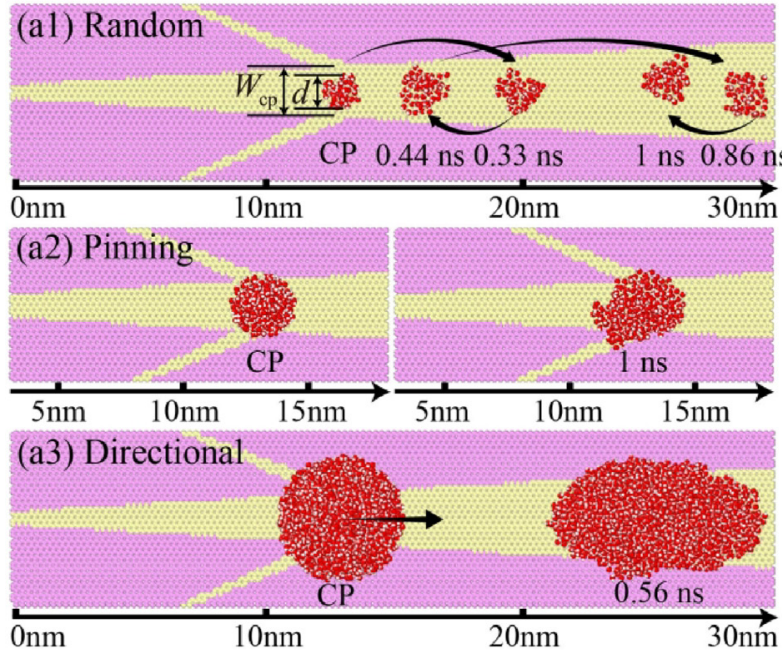

(a)

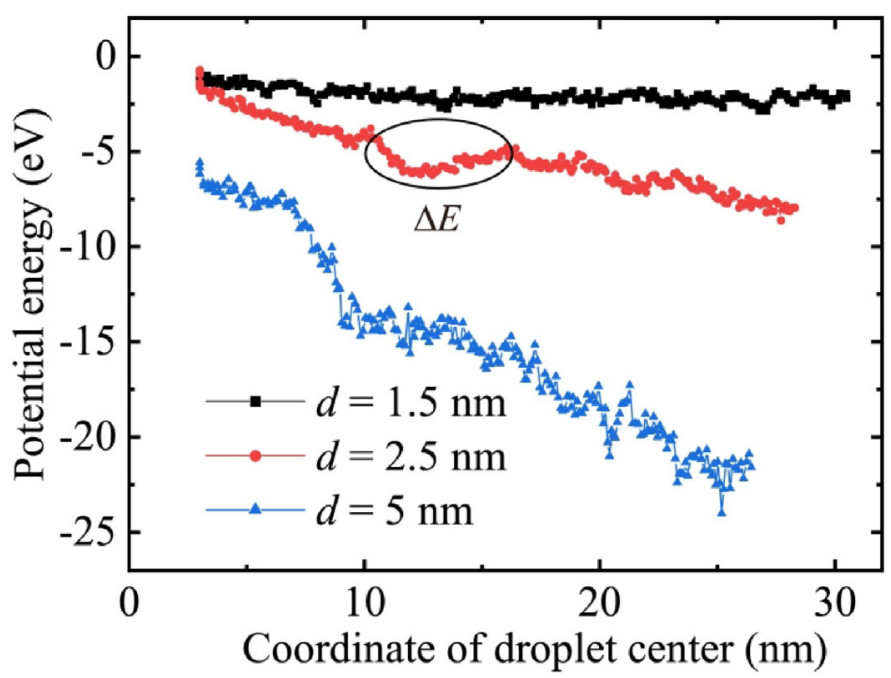

(b)

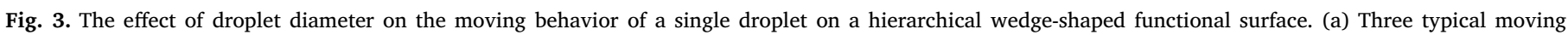

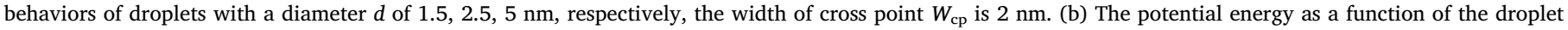
diameter. 
thermal fluctuation effect of macroscopic droplets. Such a finding should be helpful for the design of functional surfaces for faster and more efficient water collection in practice.

The above size-dependent moving behavior is further explained by the change in potential energy of droplets at different positions as shown in Fig. 3b, where the potential energy of droplets of different diameters as a function of the position of droplets is given. It is found that the potential energy of the small droplet with a diameter of $1.5 \mathrm{~nm}$ keeps almost constant with a little fluctuation, indicating a negligible driving force. Such a result is consistent with the previous study [25] that a droplet immersed in a hydrophilic or hydrophobic region would not suffer a driving force but thermal fluctuation [41], inducing Brownian-like random motion. Similar phenomena have also been observed for other nanoscale objects, such as single-molecule [42], cluster [43], nanoparticle [41,44] and nanodroplet [45]. For the medium-sized droplet with a diameter of $2.5 \mathrm{~nm}$, the potential energy decreases with the position far away from the wedge tip, but an obvious energy barrier $\Delta E$ labeled by a black cycle exists at the cross point position as shown in Fig. 3b. Here, the potential energy of a droplet at each point is achieved by placing the droplet directly at that point. If a droplet is placed at the cross point, it needs to overcome the energy barrier to move, otherwise it will be pinned. Sometimes, the limited thermal fluctuation energy is not enough to overcome the barrier. If a droplet is released at a position near the cross point, the droplet may also be pinned, similar to the case shown in Fig. 3a2. However, if a droplet is released at a position far away from the cross point, enough kinetic energy may help the droplet get over the energy barrier and keep going. An example is illustrated in Fig. S2 in Supporting information. For the large droplet with a diameter of $5 \mathrm{~nm}$, the potential energy decreases monotonically as the distance from the wedge tip increases, which is conducive to the directional motion of the droplet along the main trunk. Therefore, for a two-level wedge-shaped functional surface, on the one hand, the second-level branches can help small droplets gather on the first-level main trunk to form a large droplet and continue to move directionally; on the other hand, it also increases the hydrophilic area at the cross point and hinders the movement of droplets. Such a result puts forward higher requirements for the design of hierarchical wedge-shaped functional surface: on the one hand, small droplets from branches can converge on the main trunk, and on the other hand, the hydrophilic area at the cross point cannot be increased too much.

Inspired by the above problems, we further investigate the effect of the second-level wedge-shaped branch on the spontaneous droplet aggregation behavior, including the wedge angle, wedge direction and the symmetry of the hierarchical structure as shown in Fig. 4.

The effect of the wedge angle in the second-level structure is given in Fig. 4a, where cases of two wedge angles of $3.4^{\circ}$ and $8.7^{\circ}$ are studied as shown in Fig. 4a1 and a2, respectively. Three droplets are initially placed at the second-level and the main paths. Once all droplets are released, they spontaneously move towards the cross point and then aggregate with each other at about $0.11 \mathrm{~ns}$. Afterwards, the aggregated droplet moves further and reaches the right boundary at $0.33 \mathrm{~ns}$ in the case with a wedge angle of $3.4^{\circ}$ as shown in Fig. 4a1. Nevertheless, in the case with a wedge angle of $8.7^{\circ}$ as shown in Fig. 4a2, the aggregated droplet will be tightly pinned at the cross point. Such different behaviors caused by different wedge angles can be explained by the comparison of potential energy at different positions as shown in Fig. $4 \mathrm{a} 3$. In the case with an angle of $3.4^{\circ}$, the further away from the tip of the main trunk, the potential energy monotonically decreases. In the case of a slightly larger angle, i.e. $8.7^{\circ}$, the potential energy near the cross point has a larger energy barrier. Even if the aggregated droplet has some kinetic energy, it does not overcome the energy barrier and stops at the cross point.

The effect of bifurcation (the second-level branch) orientation on the droplet aggregation and moving behaviors is shown in Fig. 4b, in which cases of forward bifurcation and backward bifurcation are considered as shown in Fig. $4 \mathrm{~b} 1$ and b2, respectively. In both cases, the droplet has the same volume and the branch has the same wedge angle. It shows that spontaneous droplet aggregation and subsequent motion successfully happen in the forward bifurcation case while motion after aggregation fails in the backward bifurcation case. It is very interesting to find that the potential energy at each point in two respective systems is nearly the same as shown in Fig. 4b3. Specially, an energy barrier exits near the cross point. What causes these two different phenomena? The velocity of the aggregated droplet is further analyzed. In the former case, the velocity of the aggregated droplet is $104.7 \mathrm{~m} / \mathrm{s}$ near the cross point and has a forward direction, while in the latter it is $-15.3 \mathrm{~m} / \mathrm{s}$ with a backward direction.

The effect of bifurcation symmetry on the droplet aggregation and motion is given in Fig. 4c, where symmetrical distribution and alternant distribution of the second-level structures along the main trunk are analyzed. It is easy to find that aggregated droplet may be pinned at the cross point in the symmetric distribution case, while subsequent motion after aggregation successfully happens in the alternant distribution case, as shown in Fig. 4c1 and $\mathrm{c} 2$, respectively. The distribution of potential energy along the main trunk is further achieved for the two cases as shown in Fig. 4c3. It is easy to find that a relatively large energy barrier $\Delta E_{\mathrm{c} 1}$ exists in the symmetric distribution case, which should be responsible for the failure of subsequent motion.

The effect of hydrophobic defect in hydrophilic paths and shape gradient of hydrophilic paths on the spontaneous droplet aggregation and motion is further investigated, in which the hydrophobic defect is assumed to be circular as shown in Fig. 5a, b and the hydrophilic path has no shape gradient, i.e., a rectangular one is shown in Fig. 5c. A relatively small circular defect and a relatively large one are considered, the results of which are shown in Fig. 5a and b, respectively. All small droplets are placed near the wedge tips of the second-level paths and the main trunk. In Fig. 5a, it is found that three droplets on the left of the relatively small defect will aggregate and further pass through the defect. Two droplets on the right of the small defect will aggregate too. Finally, both aggregated droplets will merge and move on as shown in Fig. 5a. However, a relatively large hydrophobic defect in the main trunk will prevent the two aggregated droplets from merging as shown in Fig. 5b. Therefore, in practical applications, it is better to avoid the introduction of defects in transport paths.

It should be noted that both droplet aggregation and motion depend on the unbalanced force, which has been analytically analyzed in $[46,47]$. Shape gradient is a necessary condition for such a functional surface. Of course, some other functional surfaces could be designed through wettability gradient [34], but which are difficult to fabricate. In order to emphasize the condition of shape gradient, we also simply give the result without shape gradient as shown in Fig. 5c. Even though it is a two-level structure, droplets on the rectangular hydrophilic paths do not move and only undergo thermal oscillations.

Based on the above study of spontaneous droplet aggregation and motion on a two-level functional surface, a three-level wedge-shaped hierarchical functional surface is designed as shown in Fig. 6. The threelevel functional surface looks like a tree root. Both sides of the trunk branch into several secondary branches, which further branch into smaller branches. All branches at the same-level have a forward and asymmetric arrangement. Such a hierarchical wedge-shaped functional surface should have high water collection efficiency, the mechanism of which can be found from the water collecting behavior in a local area. Snapshots at some typical steps are shown in Fig. 6. Initially, four droplets labeled as "1", " 2 ", "3" and " 4 " will spontaneously transport towards the main trunk and aggregate to form a larger moving droplet " $1 \sim 4$ " at 0.05 ns. Meanwhile, droplets " 5 " and "6" move randomly to the main trunk and meet droplet " $1 \sim 4$ ". A new droplet " $1 \sim 6$ " forms and is pinned at a cross point at $0.1 \mathrm{~ns}$. When the droplet "7" converges with droplet " $1 \sim 6$ ", a larger droplet " $1 \sim 7$ " forms and moves forward at $0.2 \mathrm{~ns}$. At the same time, droplet " 10 " comes close to the main trunk. Finally, a behavior of "moving-pinned-converging-moving-pinnedconverging-......"occurs repeatedly, realizing efficient water collection. 
(a)

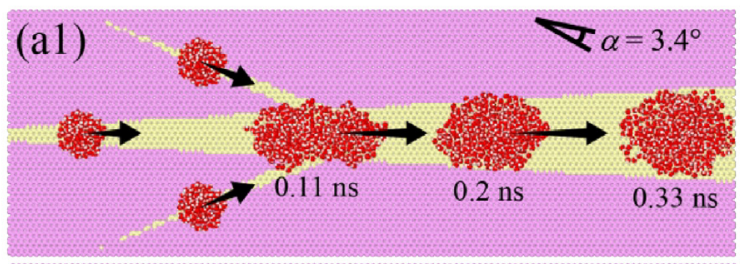

(b)
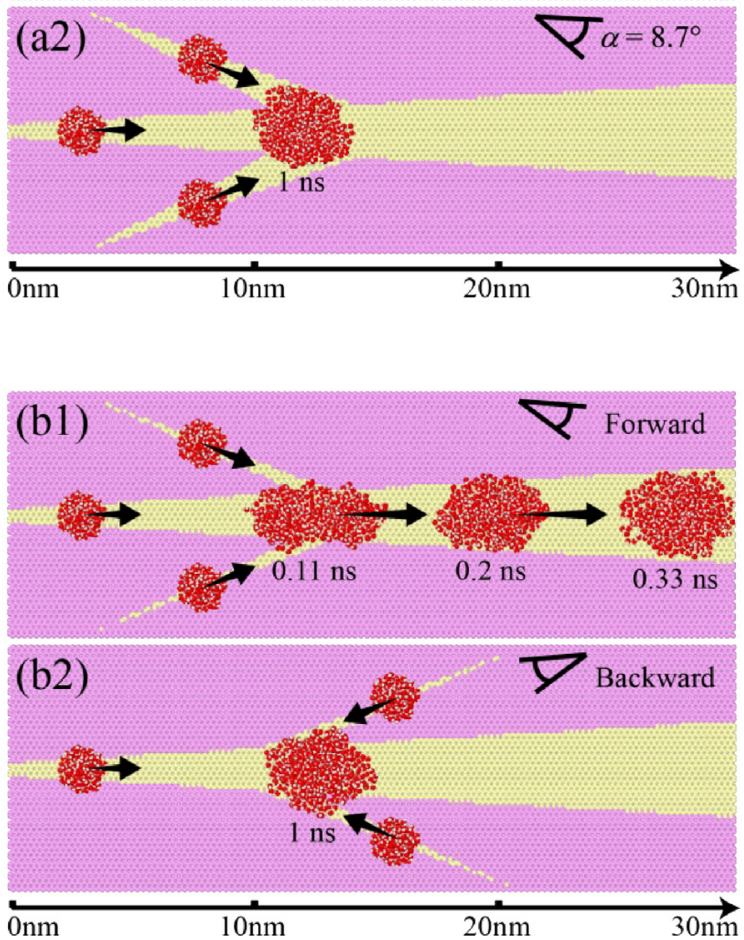

(c)
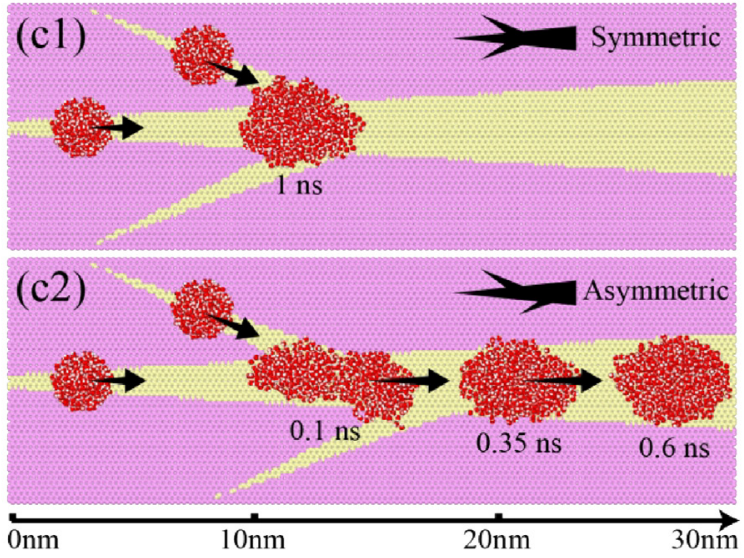
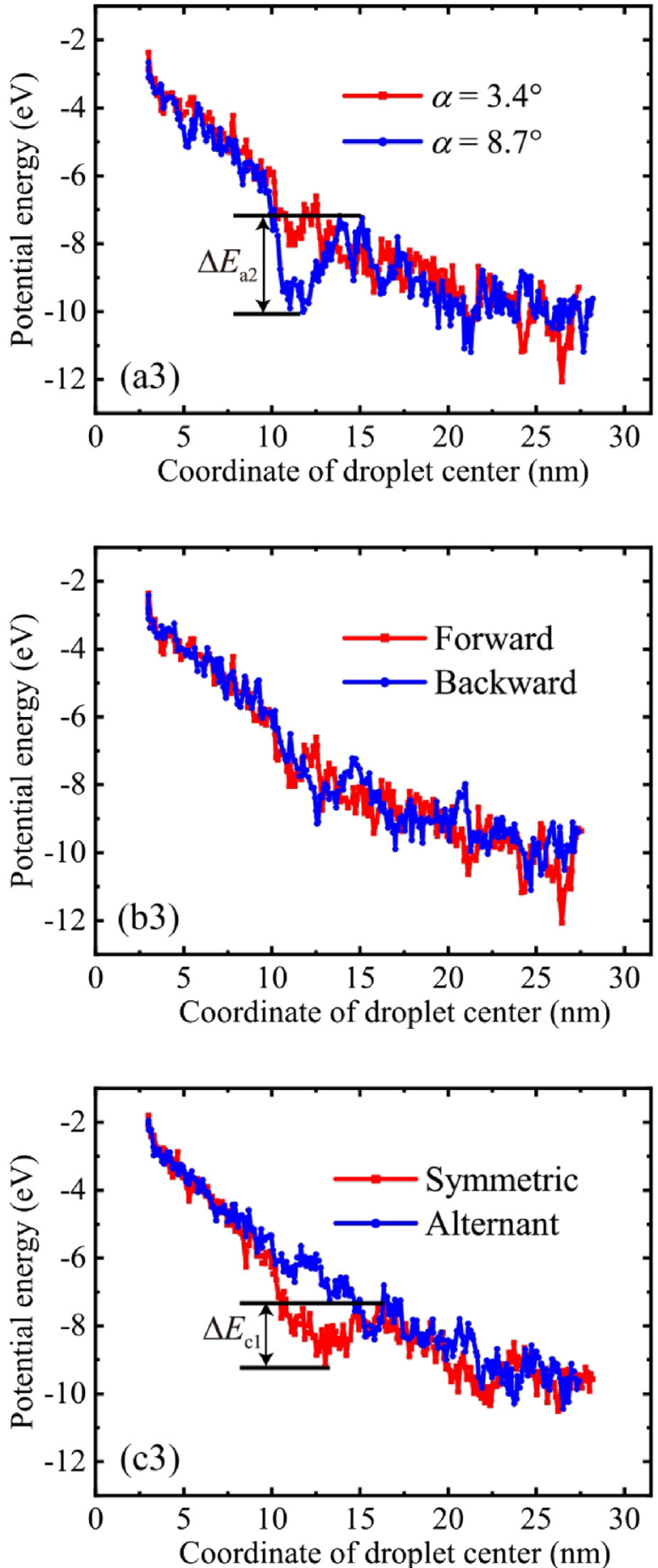

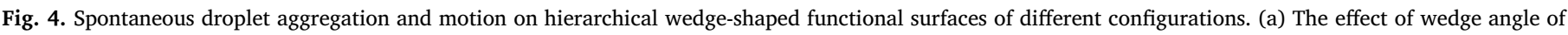
branches (second-level structure); (b) The effect of bifurcation direction; (c) The effect of bifurcation symmetry.

An intuitional demonstration of such a process is given in Movie S3 in Supporting Information.

\section{Conclusion}

Based on molecular dynamics simulations, numerical experiments are systematically carried out in order to study the influencing factors of spontaneous water collection on a hierarchical wedge-shaped functional surface. Several important influencing factors, such as the volume of droplet, wedge angle, wedge orientation and symmetry of eachlevel bifurcation, are systematically analyzed. Typical behaviors of droplet aggregation and motion are found and explained from the potential energy point of view. It is found that three main conditions need to be met: small wedge angles at each level, same orientation at eachlevel and different levels and asymmetric branches at the same level. A three-level wedge-shaped hierarchical functional surface is further numerically designed, which can realize efficient water collection. The results in this paper should be helpful for the precise design of hierarchical functional surfaces of water collection in practical application. 

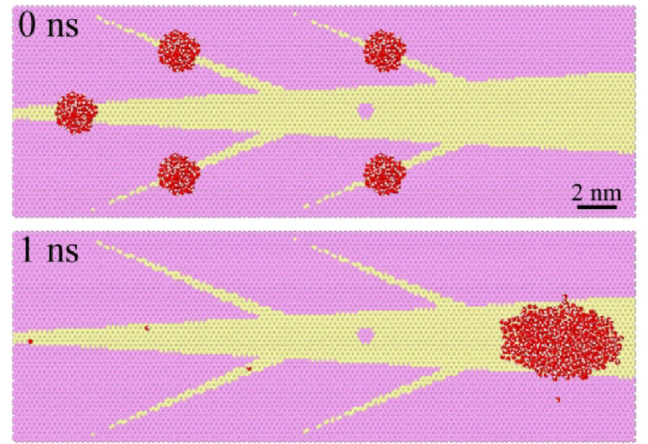

(a)
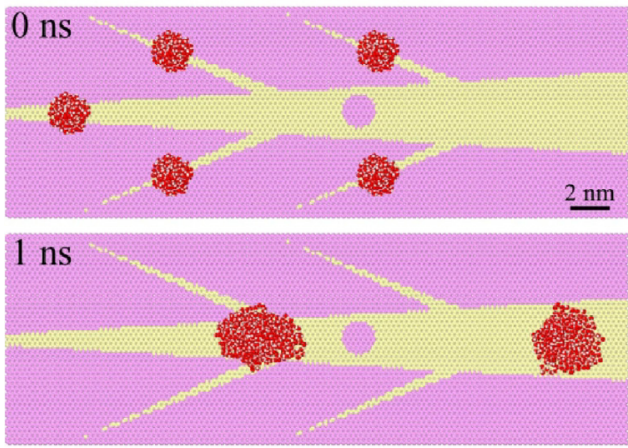

(b)
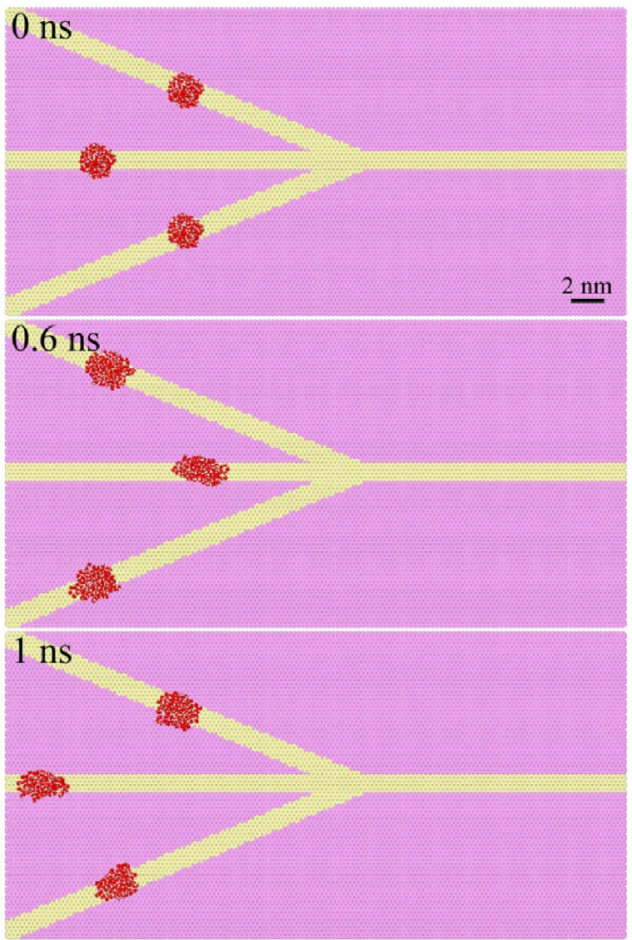

(c)

Fig. 5. The effect of hydrophobic defect and shape gradient on the spontaneous droplet aggregation and motion. (a) A circular hydrophobic defect with a diameter of $0.8 \mathrm{~nm}$; (b) A circular hydrophobic defect with a diameter of $1.6 \mathrm{~nm}$; (c) Surface consisting of rectangular hydrophilic strips.

\section{Data availability}

The raw/processed data required to reproduce these findings cannot be shared at this time as the data also forms part of an ongoing study.

\section{CRediT authorship contribution statement}

Shuai Wang: Methodology, Software, Validation, Formal analysis, Investigation, Data curation, Writing - original draft, Supervision. Zhilong Peng: Writing - review \& editing, Supervision, Project administration. Jianjun Li: Writing - review \& editing. Yazheng Yang: Writing - review \& editing. Chao Wang: Conceptualization, Methodology, Writing - review \& editing, Validation, Supervision, Project administration. Shaohua Chen: Conceptualization, Resources, Writing - review \& editing, Visualization, Supervision, Project administration, Funding acquisition.
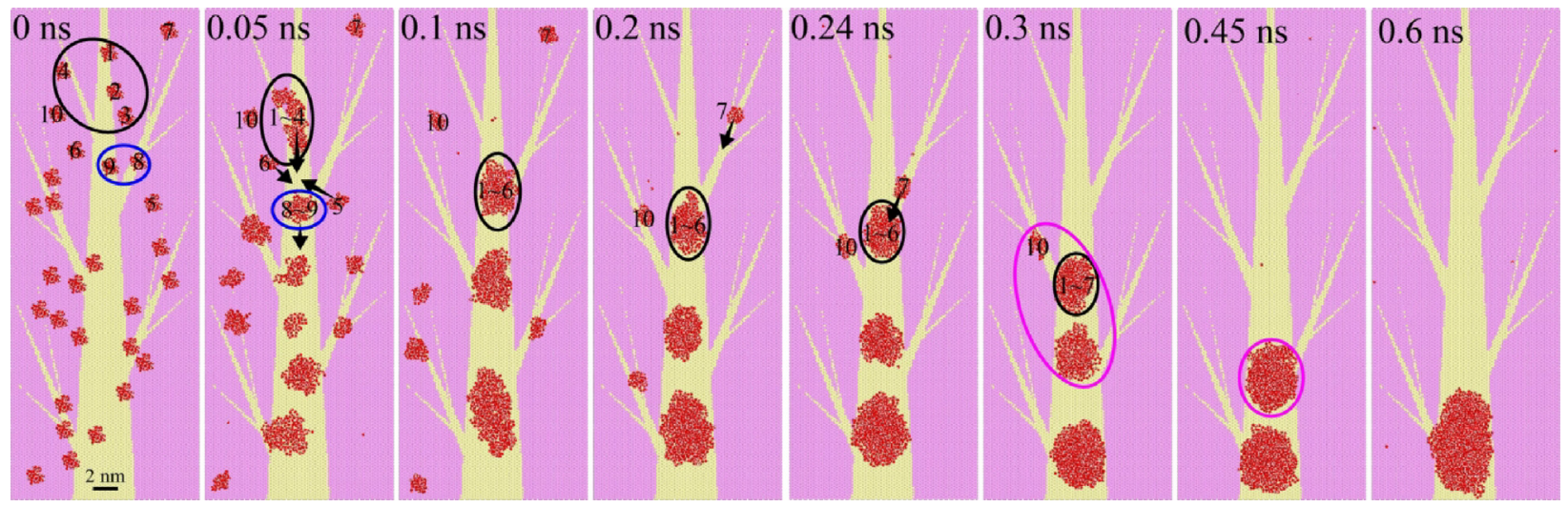

Fig. 6. Snapshots of spontaneous water collection on a three-level hierarchical wedge-shaped functional surface. Wedge angles of the main trunk, secondary branches and the smallest ones are $6.54^{\circ}, 3.41^{\circ}$ and $2.14^{\circ}$, respectively. 


\section{References}

[1] S.C. Hernandez, C.J. Bennett, C.E. Junkermeier, S.D. Tsoi, F.J. Bezares, R. Stine, J.T. Robinson, E.H. Lock, D.R. Boris, B.D. Pate, ACS Nano 7 (2013) 4746-4755.

[2] S.Y. Teh, R. Lin, L.H. Hung, A.P. Lee, Lab. Chip 8 (2008) 198-220.

[3] J. Atencia, D.J. Beebe, Nature 437 (2005) 648-655.

[4] H. Chen, P. Zhang, L. Zhang, H. Liu, Y. Jiang, D. Zhang, Z. Han, L. Jiang, Nature 532 (2016) 85.

[5] J.K. Domen, W.T. Stringfellow, M.K. Camarillo, S. Gulati, Clean Technol. Environ. Pol. 16 (2014) 235-249.

[6] J.M. Wigzell, R.C. Racovita, B.G. Stentiford, M. Wilson, M.T. Harris, I.W. Fletcher, D.P.K. Mosquin, D. Justice, S.K. Beaumont, R. Jetter, J.P.S. Badyal, Colloids Surf. A 506 (2016) 344-355.

[7] A.R. Parker, C.R. Lawrence, Nature 414 (2001) 33-34.

[8] H. Bai, X. Tian, Y. Zheng, J. Ju, Y. Zhao, L. Jiang, Adv. Mater. 22 (2010) 5521-5525.

[9] Y. Zheng, H. Bai, Z. Huang, X. Tian, F.-Q. Nie, Y. Zhao, J. Zhai, L. Jiang, Nature 463 (2010) 640.

[10] L. Zhang, J. Wu, M.N. Hedhili, X. Yang, P. Wang, J. Mater. Chem. A 3 (2015) 2844-2852.

[11] A. Lee, M.-W. Moon, H. Lim, W.-D. Kim, H.-Y. Kim, Langmuir 28 (2012) 10183-10191.

[12] H. Bai, L. Wang, J. Ju, R. Sun, Y. Zheng, L. Jiang, Adv. Mater. 26 (2014) 5025-5030.

[13] S. Choo, H.-J. Choi, H. Lee, Appl. Surf. Sci. 324 (2015) 563-568.

[14] K. Yin, H. Du, X. Dong, C. Wang, J.-A. Duan, J. He, Nanoscale 9 (2017) 14620-14626.

[15] M.K. Chaudhury, G.M. Whitesides, Science 256 (1992) 1539-1541.

[16] Z. Wang, M. Elimelech, S. Lin, Environ. Sci. Technol. 50 (2016) 2132-2150.

[17] A.P. Malshe, S. Bapat, K.P. Rajurkar, H. Haitjema, CIRP Ann. 67 (2018) 627-650.

[18] D.-J. Huang, T.-S. Leu, J. Micromech. Microeng. 25 (2015) 085007.

[19] J. Zhang, Y. Han, Langmuir 23 (2007) 6136-6141.

[20] A. Ghosh, R. Ganguly, T.M. Schutzius, C.M. Megaridis, Lab. Chip 14 (2014) $1538-1550$.

[21] H.S. Khoo, F.-G. Tseng, Appl. Phys. Lett. 95 (2009) 063108.

[22] T. Ody, M. Panth, A. Sommers, K. Eid, Langmuir 32 (2016) 6967-6976.

[23] M. Alheshibri, N. Rogers, A. Sommers, K. Eid, Appl. Phys. Lett. 102 (2013) 174103.

[24] Y. Zheng, J. Cheng, C. Zhou, H. Xing, X. Wen, P. Pi, S. Xu, Langmuir 33 (2017)
$4172-4177$.

[25] S. Wang, C. Wang, Z. Peng, S. Chen, J. Phys. Chem. C 123 (2019) 1798-1805.

[26] M. Wang, Q. Liu, H. Zhang, C. Wang, L. Wang, B. Xiang, Y. Fan, C.F. Guo, S. Ruan, ACS Appl. Mater. Interfaces 9 (2017) 29248-29254.

[27] J. Lin, X. Tan, T. Shi, Z. Tang, G. Liao, ACS Appl. Mater. Interfaces 10 (2018) 44815-44824.

[28] W. Liu, P. Fan, M. Cai, X. Luo, C. Chen, R. Pan, H. Zhang, M. Zhong, Nanoscale 11 (2019) 8940-8949.

[29] H. Chen, L. Zhang, P. Zhang, D. Zhang, Z. Han, L. Jiang, Small 13 (2017) 1601676.

[30] M.A. González, J.L. Abascal, J. Chem. Phys. 132 (2010) 096101.

[31] H.J.C. Berendsen, J.R. Grigera, T.P. Straatsma, J. Phys. Chem. 91 (1987) 6269-6271.

[32] J.P. Ryckaert, G. Ciccotti, H.J.C. Berendsen, J. Comput. Phys. 23 (1977) 327-341.

[33] R.W. Hockney, J.W. Eastwood, Computer Simulation Using Particles, CRC Press, 1988.

[34] O. Liu, B. Xu, Langmuir 31 (2015) 9070-9075.

[35] C.J. Lv, C. Chen, Y.-C. Chuang, F.-G. Tseng, Y.J. Yin, F. Grey, Q.S. Zheng, Phys. Rev. Lett. 113 (2014) 026101.

[36] A. Ashraf, Y. Wu, M.C. Wang, K. Yong, T. Sun, Y. Jing, R.T. Haasch, N.R. Aluru, S. Nam, Nano Lett. 16 (2016) 4708-4712.

[37] N. Wei, C. Lv, Z. Xu, Langmuir 30 (2014) 3572-12578.

[38] Q.S. Zheng, C.J. Lv, P.F. Hao, J. Sheridan, Science China Physics, Mech. Astron. 53 (2010) 2245-2259.

[39] W. Shinoda, M. Shiga, M. Mikami, Phys. Rev. B 69 (2004) 134103.

[40] S. Plimpton, J. Comput. Phys. 117 (1993) 1-19.

[41] M. Jafaryzadeh, C.D. Reddy, V. Sorkin, Y.W. Zhang, Nanoscale Res. Lett. 7 (2012) 1-8.

[42] H. Hedgeland, P. Fouquet, A.P. Jardine, G. Alexandrowicz, W. Allison, J. Ellis, Nat. Phys. 5 (2009) 561.

[43] M. Kim, S.M. Anthony, S. Granick, Phys. Rev. Lett. 102 (2009) 178303.

[44] A. Schirmeisen, Nat. Mater. 9 (2010) 615.

[45] Z.-X. Niu, T. Huang, Y. Chen, Front. Phys. 13 (2018) 137804.

[46] M. Liu, L. Huang, Y. Yao, Z. Peng, S. Chen, J. Phys. Chem. C 123 (2019) 23505-23510.

[47] M. Liu, Y. Yao, Y. Yang, Z. Peng, S. Chen, J. Phys. Chem. C 123 (2019) 12736-12743. 